\title{
GLOBAL ATTRACTIVITY AND STABILITY IN SOME MONOTONE DISCRETE DYNAMICAL SYSTEMS
}

\author{
XIAO-QIANG ZHAO
}

\begin{abstract}
The existence of globally attractive order intervals for some strongly monotone discrete dynamical systems in ordered Banach spaces is first proved under some appropriate conditions. With the strict sublinearity assumption, threshold results on global asymptotic stability are then obtained. As applications, the global asymptotic behaviours of nonnegative solutions for time-periodic parabolic equations and cooperative systems of ordinary differential equations are discussed and some biological interpretations and concrete application examples are also given.
\end{abstract}

\section{INTRODUCTION}

Recently both continuous and discrete-time strongly order-preserving dynamical systems have been extensively investigated and developed. (See $[5,7,13,14,15$, 16, 19, 20, 21, 22, 23, 25] and references therein.) Now it is known that typical asymptotic behavior in strongly monotone dynamical systems is generic convergence to an equilibrium in the continuous-time case $($ see $[16,20,23])$ and to cycles in the discrete- time case (see $[\mathbf{2 1}]$ ) for "almost all" relatively compact orbits. From the practical point of view, one desires a more complete description on the asymptotic behaviors of all orbits, for example, convergence everywhere, global attractor and global stability of equilibrium. This of course requires some additional restrictions on the monotone dynamical systems considered. For discrete strongly monotone dynamical systems on the finite dimensional ordered space $\left(R^{n}, R_{+}^{n}\right)$, which can be generated by periodic cooperative and irreducible systems of ordinary differential equations, Smith [22] essentially obtained threshold results on the global attractivity of either positive fixed points or zero fixed points under strong concavity assumptions. In [25], Takác introduced the notion of sublinearity of $S$ on $V \subset E$, that is, for any $\alpha \in[0,1], u \in$ $V, S(\alpha u) \geqslant \alpha S(u)$, and studied the convergence of all orbits in $V$ and the stability of

Received 14th June, 1995

Research supported by the National Science Foundation of People's Republic of China. The author would like to express his gratitude to Professor E.N. Dancer for his invaluable advice and discussion on quasilinear periodic-parabolic problems and monotone dynamical systems. The author is also grateful to Professor K. Gopalsamy for his helpful discussions on the global asymptotic behaviours in population dynamics.

Copyright Clearance Centre, Inc. Serial-fee code: 0004-9729/96 \$A2.00+0.00. 
fixed points of $S$. (See also [13, Chapter I.5].) As for the convergence problem, we also refer to Hale and Raugel's recent work on gradient-like dynamical systems in [10], and references therein.

In this paper we mainly study global attractivity and global asymptotic stability in discrete strongly monotone dynamical systems on an ordered Banach space $E$. In Section 2, based on the Krein-Rutman theorem, we first prove the existence of a globally attractive order interval with two fixed points of $S$ as its endpoints and a stable fixed point of $S$ inside, either between two linearly unstable fixed points of $S$ or between a linearly unstable fixed point and a superequilibrium of $S$ (Theorem 2.1). Then we prove the global asymptotic stability of the linearly stable or linearly neutrally stable fixed point $0=S(0)$ in a positively invariant order interval $V=[0, b]_{E}$ and the cone $P$ of $E$ (Theorem 2.2) under the assumption that

$\left(H_{0}\right) S(u)<D S(0) u$ for all $u \in V$ with $u \gg 0$.

We further introduce the notion of strict sublinearity of $S$ on $V$, that is,

$$
S(\alpha u)>\alpha S(u) \quad \text { for all } \alpha \in(0,1) \text { and } u \in V \text { with } u \gg 0,
$$

which implies the uniqueness of positive fixed point in $V$ and $\left(H_{0}\right)$ when $S(0)=0$ (Lemma 1). As a consequence of Theorems 1 and 2, we obtain threshold results on the global asymptotic stability of either a positive fixed point or zero fixed point on $V=[0, b]$ or $V=P$ under the assumption that $S^{m}$ is strictly sublinear on $V$ for some integer $m \geqslant 1$ (Theorems 2.3 and 2.4). Theorems 2.3 and 2.4 generalise Hirsch [14, Theorem 6.1] and Smith's [22, Theorems 2.1-2.3] results in the finite dimensional ordered space $\left(R^{n}, R_{+}^{n}\right)$ to the infinite dimensional ordered Banach space $(E, P)$ with the strict sublinearity assumption much weaker than their strong concavity ones (Remark 2.2), and also strengthen Takáć's result [25], (Remark 2.3). Morever, according to the remarks in [8], our standard assumption that $S: V \rightarrow V$ is compact can be actually replaced by a weaker one: $S: V \rightarrow V$ is condensing and $S\left([u, v]_{E}\right)$ is bounded in $E$ for all $u, v \in V$ with $u<v$ (Remark 2.4).

In Section 3, we first apply the abstract theorems in Section 2 to the Poincaré operator defined by periodic parabolic equations with reaction term $f(x, t, u)=u F(x, t, u)$, and obtain corresponding results on the global asymptotic behavior of positive solutions and the existence of at least one stable positive periodic solution (Theorem 3.1), global asymptotic stability of a zero solution (Theorem 3.2) and global asymptotic stability of a positive periodic solution (Theorem 3.3), which lead to a threshold criterion for the "uniform persistence" and "extinction" of a single species population. Theorem 3.3 also implies Hess's result [13, Theorem 28.11] for the periodic-parabolic Logistic equations. Furthermore, as a consequence of Theorems 3.2 and 3.3, we obtain a threshold result for autonomous parabolic equations (Theorem 3.4), which generalises 
Cantrell and Cosner's results on the global asymptotic stability of positive steady-state solution in the Dirichlet boundary condition case (see [3, Theorems 2.1 and 2.3] and [4, Theorem 2.4 with Corollary 3.3]). As an application example of Theorem 3.4, for a reaction-diffusion equation deduced from the competition model in an unstirred chemostat (see $[\mathbf{1 7}, \mathbf{2 4}]$ ) we get a threshold result on the global asymptotic stability of either a positive steady-state solution or zero steady-state solution (Proposition), which generalises Hsu and Waltman's recent similar result for the Michaelis-Menten-Monod function $F=(m s) /(a+s), s \geqslant 0[17$, Theorems 3.1 and 3.2] to a more general function $F$ satisfying $F(0)=0$ and $F^{\prime}(s)>0$ for $s \geqslant 0$. Finally we show how the abstract results in Section 2 can be applied to periodic cooperative and irreducible systems of ordinary differential equations and, as an illustration of Theorem 2.4, give a threshold result on the global asymptotic stability of either a positive periodic solution or zero solution (Theorem 3.5). An application example of Theorem 3.5 to the single loop positive feedback systems in $R_{+}^{n}$ is also given under the strict sublinearity assumption on $f(x, t)$ instead of the strong concaivity assumption in [22].

\section{Global attractivity and STABILITY}

Let $(E, P)$ be an ordered Banach space with positive cone $P$ having nonempty interior $\operatorname{int}(P)$. For $x, y \in E$, we write

$$
\begin{array}{ll}
x \geqslant y & \text { if } x-y \in P \\
x>y & \text { if } x-y \in P \backslash\{0\} \\
x \gg y & \text { if } x-y \in \text { int }(P) .
\end{array}
$$

Let $U$ be an open subset of $E$. In this section, we always assume $S: U \rightarrow U$ is a continuous and strongly order-preserving mapping, that is, $x, y \in U, x>y$ implies $S(x) \gg S(y)$. If $u \in U$ and $S$ is Fréchet differentiable at $u$, we denote this derivative by $D S(u)$.

Let $a \ll b \in E$, and $V=[a, b]_{E}$, the order interval in $E$, or alternatively, let $V=P$. In what follows, we shall assume that

(H) $V \subset U$ and $S: V \rightarrow V$ is order-compact, that is, $S\left([u, v]_{E}\right)$ is relatively compact for all $u, v \in V$ with $u<v$.

Let $K \in L(E)$ be a linear operator. We call $K$ strongly positive if $K(P \backslash\{0\}) \subset$ $\operatorname{int}(P)$. If $K$ is compact and strongly positive, we denote its spectral radius by $r(K)$, the existence and positivity of which are guaranteed by the Krein-Rutman theorem (see, for example, [13, Theorem 7.2]).

We first prove the following result. 
THEOREM 2.1. Let $V=[a, b]_{E}$ with $a \ll b$ and let $(H)$ hold. Assume that

(1) $S(a)=a, D S(a)$ exists and $D S(a)$ is compact, strongly positive, and $r(D S(a))>1$;

(2) either $S(b)=b, D S(b)$ exists and $D S(b)$ is compact, strongly positive, and $r(D S(b))>1$, or alternatively, $S(b) \leqslant b$.

Then there exist two fixed points $u_{1}$ and $u_{2}$ of $S$ in $[a, b]_{E}$ with $u_{2} \geqslant u_{1} \gg a$ such that for any $u \in(a, b)_{E}=\{u \in E ; a<u<b\}$,

$$
\lim _{n \rightarrow \infty} \operatorname{dist}_{E}\left(S^{n}(u),\left[u_{1}, u_{2}\right]\right)=0
$$

and $\left[u_{1}, u_{2}\right]$ contains a stable fixed point $u_{0}$ of $S$. Morever, $u_{2} \ll b$ in the case either $S(b)=b$ and $r(D S(b))>1$ or $S(b)<b$.

Proof: We prove the theorem under the first assumption of (2). For the second case, a similar proof works.

By the Krein-Rutman Theorem [13, Chapter I.7], let $e_{1} \gg 0, e_{2} \gg 0,\left\|e_{i}\right\|_{E}=1$ be the principal eigenfunctions of $D S(a)$ and $D S(b)$ respectively. Then

$$
\begin{array}{ll}
D S(a) e_{1}=r_{1} e_{1}, & r_{1}=r(D S(a)) \\
D S(b) e_{2}=r_{2} e_{2}, & r_{2}=r(D S(b)) .
\end{array}
$$

Since $r_{1}>1, r_{2}>1$ and for $\varepsilon>0$

$$
\begin{aligned}
S\left(a+\varepsilon e_{1}\right) & =S(a)+D S(a)\left(\varepsilon e_{1}\right)+o(\|\varepsilon\|) \\
& =a+\varepsilon r_{1} e_{1}+o(\|\varepsilon\|) \\
S\left(b-\varepsilon e_{2}\right) & =S(b)+D S(b)\left(-\varepsilon e_{2}\right)+o(\|\varepsilon\|) \\
& =b-\varepsilon r_{2} e_{2}+o(\|\varepsilon\|),
\end{aligned}
$$

there exists $\varepsilon_{0}>0$ such that for any $0<\varepsilon \leqslant \varepsilon_{0}$,

$$
S\left(a+\varepsilon e_{1}\right)>a+\varepsilon e_{1} \text { and } S\left(b-\varepsilon e_{2}\right)<b-\varepsilon e_{2},
$$

that is, $a+\varepsilon e_{1}<b-\varepsilon e_{2}$ are order-related sub- and superequilibria of $S$. Therefore, by [13, Lemma 1.1], the set $F=\{u ; S(u)=u, a<u<b\} \neq \emptyset$. We claim that $F$ is compact. Indeed, since $F=S(F) \subset S([a, b])$ is relatively compact, it suffices to prove that $F$ is a closed subset of $E$. For any $u \in F$, since $S$ is strongly orderpreserving, $a \ll u \ll b$. Assume $u_{n} \in F(n=1,2, \cdots), u_{n} \rightarrow u(n \rightarrow \infty)$. Then $\left\|u_{n}-a\right\|_{E}>0,\left\|u_{n}-b\right\|_{E}>0$. Morever, $a \leqslant u \leqslant b$ and, by the continuity of $S$, $S(u)=u$. Now assume $u=a$. Then

$$
u_{n}-a=S\left(u_{n}\right)-S(a) \stackrel{\leftrightarrow}{=} S(a)\left(u_{n}-a\right)+o\left(\left\|u_{n}-a\right\|\right) .
$$


Let $v_{n}=\left(u_{n}-a\right) /\left(\left\|u_{n}-a\right\|\right) \gg 0$, then

$$
v_{n}=D S(a) v_{n}+\frac{o\left(\left\|u_{n}-a\right\|\right)}{\left\|u_{n}-a\right\|} \text {. }
$$

Since $D S(a)$ is compact and $\left\|v_{n}\right\|=1$, we may assume, without loss of generality, that $D S(a) v_{n}$ converges. Hence $v_{n}$ converges to $v$ say, where $\|v\|_{E}=1, v>0$, and $v=D S(a) v$. By the Krein-Rutman theorem, $r(D S(a))=1$, which contradicts the assumption $r(D S(a))>1$. Therefore $u \neq a$. By a similar proof, $u \neq b$. Then $u \in F$, and hence $F$ is a closed set.

From the compactness of $F$ it follows that there exists an $\varepsilon_{0}>0$ such that for any $u \in F, a+\varepsilon_{0} e_{1}<u<b-\varepsilon_{0} e_{2}$, and for any $\varepsilon \in\left(0, \varepsilon_{0}\right], a+\varepsilon e_{1}<b-\varepsilon e_{2}$ are order-related strict sub- and superequilibria. From the Dancer-Hess Theorem (see [7, Theorem 3] or $\left[13\right.$, Theorem 4.2]) it follows that there exists a stable fixed point $u_{0} \in\left[a+\varepsilon e_{1}, b-\varepsilon e_{2}\right]$. Further by [13,Lemma 1.1], $S^{n}\left(a+\varepsilon e_{1}\right)$ converges increasingly to the minimal fixed point $u_{1}$ of $S$ and $S^{n}\left(b-\varepsilon e_{2}\right)$ converges decreasingly to the maximal fixed point $u_{2}$ of $S$ in the interval $\left[a+\varepsilon e_{1}, b-\varepsilon e_{2}\right]$. We further claim that $u_{1}$ and $u_{2}$ are independent of the choice of $\varepsilon \in\left(0, \varepsilon_{0}\right]$. Indeed, for any $\varepsilon_{1}, \varepsilon_{2} \in\left(0, \varepsilon_{0}\right]$, let $u_{1}^{(i)} \leqslant u_{2}^{(i)}(i=1,2)$ be the corresponding minimal and maximal fixed points with $\varepsilon=\varepsilon_{i}$ respectively. Since $u_{i}^{(j)} \in F(i=1,2, j=1,2)$, by the choice of $\varepsilon_{0}$ above,

$$
a+\varepsilon_{j} e_{1} \leqslant a+\varepsilon_{0} e_{1}<u_{1}^{(i)} \leqslant u_{2}^{(i)}<b-\varepsilon_{0} e_{2} \leqslant b-\varepsilon_{j} e_{2}, i=1,2, j=1,2 .
$$

Again by $[\mathbf{1 3}$, Lemma 1.1],

$$
u_{1}^{(j)} \leqslant u_{1}^{(i)} \leqslant u_{2}^{(i)} \leqslant u_{2}^{(j)}, \quad i=1,2, \quad j=1,2
$$

which implies that

$$
u_{1}^{(1)}=u_{1}^{(2)}, \quad u_{2}^{(1)}=u_{2}^{(2)} .
$$

Finally, we prove that for any $u \in(a, b)$

$$
\lim _{n \rightarrow \infty} \operatorname{dist}_{E}\left(S^{n}(u),\left[u_{1}, u_{2}\right]\right)=0 .
$$

Since $\left\{S^{n}(u)\right\} \subset S([a, b])$ is relatively compact, it suffices to prove that for any $u \in$ $(a, b)$, its $\omega$-limit set $\omega(u) \subset\left[u_{1}, u_{2}\right]$. For any $u^{*} \in \omega(u)$, there exist $n_{k} \rightarrow \infty$ such that $S^{n_{k}} \rightarrow u^{*}(k \rightarrow \infty)$. Since $u \in(a, b)$ and $S$ is strongly order-preserving, $a \ll S(u) \ll b$, and hence there exists an $\varepsilon \in\left(0, \varepsilon_{0}\right)$ such that $a+\varepsilon e_{1} \leqslant S(u) \leqslant b-\varepsilon e_{2}$. Then

$$
S^{n_{k}-1}\left(a+\varepsilon e_{1}\right) \leqslant S^{n_{k}}(u) \leqslant S^{n_{k}-1}\left(b-\varepsilon e_{2}\right)
$$


which implies that $u_{1} \leqslant u^{*} \leqslant u_{2}$.

This completes the proof.

ThEOREM 2.2. Let either $V=[0, b]_{E}$ with $b \gg 0$ or $V=P$ and let $(H)$ hold. Assume that

(1) $S(0)=0, D S(0)$ is compact and strongly positive, and $r(D S(0)) \leqslant 1$;

(2) $S(u)<D S(0) u$ for any $u \in V$ with $u \gg 0$.

Then $u=0$ is globally asymptotically stable with respect to $V$.

Proof: We first show that there exists no positive fixed point of $S$ in $V$. Assume that, by contradiction, there exists $u \in V, u>0$ such that $u=S(u)$. Since $S$ is strongly order-preserving, $u \gg 0$, and hence, by assumption (2),

$$
(-u)-D S(0)(-u)=D S(0) u-S(u)>0 .
$$

By the Krein-Rutman Theorem (see [13, Theorem 7.3]), $r(D S(0))>1$, which contradicts our assumption $r(D S(0)) \leqslant 1$.

Now we let $V=P$. For $V=[0, b]_{E}$, the proof is much easier. Let $e \gg 0$ be the principal eigenfunction of $D S(0)$. Then $D S(0) e=r(D S(0)) e$, and hence for any $t>0$, by assumptions (1) and (2),

$$
S(t e)<D S(0)(t e)=t \cdot r(D S(0)) e \leqslant t e .
$$

That is, te is a strict superequilibrium of $S$. For any $u \in P$, there exists $t>0$ such that $u \in[0, t e]_{E}$. By $[13$, Lemma 1.1] and the nonexistence of a positive fixed point of $S, 0 \leqslant S^{n}(u) \leqslant S^{n}(t e) \rightarrow 0$, that is, $\lim _{n \rightarrow \infty} S^{n_{n}}(u)=0$. Since $\left\{S^{n}(t e)\right\}$ is a strictly decreasing sequence in $P$, then $u=0$ is an order stable from above fixed point of $S$ in $P$, and hence, by [7, Remark 3.2] or [13, Lemma 4.3], $u=0$ is stable with repect to $P$.

This completes the proof.

Before we show an application of Theorems 2.1 and 2.2, we give the following lemmas.

Lemma 1. Let either $V=[0, b]_{E} \subset E$ or $V=P$. Assume $S: V \rightarrow V$ is continuous, strongly order-preserving and strictly sublinear on $V$, that is, $S(\alpha u)>$ $\alpha S(u)$ for all $\alpha \in(0,1)$ and $u \in V$ with $u \gg 0$. Then $S$ admits at most one positive fixed point in $V$. If, in addition, $S(0)=0$ and $D S(0)$ exists, then $S(u)<D S(0) u$ for all $u \in V$ with $u \gg 0$.

Proof: Assume that, by contradiction, $S$ admits two positive fixed points $u_{1}$ and $u_{2}$ in $V$ and $u_{1} \neq u_{2}$. Since $u_{1}>0, u_{2}>0, S(0) \geqslant 0$ and $S$ is strongly orderpreserving, $u_{1} \gg 0, u_{2} \gg 0$. Since $u_{1} \neq u_{2}$, without loss of generality we may assume 
$u_{1} \notin\left[0, u_{2}\right]_{E}$. Let $\sigma_{0}=\sup \left\{\sigma \geqslant 0 ; \sigma u_{1} \leqslant u_{2}\right\}$. Clearly, $\sigma_{0} u_{1} \leqslant u_{2}$ and $0<\sigma_{0}<1$. In the case $\sigma_{0} u_{1}<u_{2}$, we have

$$
u_{2}=S\left(u_{2}\right) \gg S\left(\sigma_{0} u_{1}\right) \geqslant \sigma_{0} S\left(u_{1}\right)=\sigma_{0} u_{1}
$$

and hence $u_{2}-\sigma_{0} u_{1} \gg 0$, which contradicts the definition of $\sigma_{0}$. In the case $\sigma_{0} u_{1}=u_{2}$, we have

$$
\sigma_{0} u_{1}=u_{2}=S\left(u_{2}\right)=S\left(\sigma_{0} u_{1}\right)>\sigma_{0} S\left(u_{1}\right)=\sigma_{0} u_{1},
$$

which also leads to a contradiction. Therefore $S$ admits at most one positive fixed point in $V$.

For any $u \in V$ with $u \gg 0$, we have $\|u\|>0$. Since $S(0)=0$ and for any $0<\alpha<1$,

$$
\begin{aligned}
S(u)<\frac{S(\alpha u)}{\alpha} & =\frac{S(0)+D S(0)(\alpha u)+o(\|\alpha u\|)}{\alpha} \\
& =D S(0) u+\frac{o(\|\alpha u\|)}{\|\alpha u\|} \cdot\|u\|,
\end{aligned}
$$

if we let $\alpha \rightarrow 0$, it follows that $S(u) \leqslant D S(0) u$. We further show that $S(u)<D S(0) u$ for all $u \in V$ with $u \gg 0$. Indeed, assume there exists $u_{0} \in V$ with $u_{0} \gg 0$ such that $S\left(u_{0}\right)=D S(0)\left(u_{0}\right)$. Then for any $0<\alpha<1, \alpha u_{0} \gg 0$ and

$$
\alpha S\left(u_{0}\right)<S\left(\alpha u_{0}\right) \leqslant D S(0)\left(\alpha u_{0}\right)=\alpha D S(0)\left(u_{0}\right)=\alpha S\left(u_{0}\right),
$$

which is a contradiction.

This completes the proof.

From the proof of $[25$, Corollary 1.2] it follows that the following lemma is valid.

Lemma 2. Let either $V=[0, b]_{E} \subset E$ or $V=P$. Assume $S: V \rightarrow V$ is continuous, strongly order-preserving and for some $m \in \mathbb{N}, S^{m}$ is sublinear, that is,

$$
S^{m}(\alpha u) \geqslant \alpha S^{m}(u) \text { for all } \alpha \in(0,1) \text { and } u \in V \text { with } u \gg 0 .
$$

Then $F_{m}=\left\{u ; u \in V \backslash\{0\}, S^{m}(u)=u\right\}=\{u ; u \in V \backslash\{0\}, S(u)=u\}=F_{1}$.

Now we are in a position to prove the following threshold result.

Theorem 2.3. Let $V=[0, b]_{E}$ with $b \gg 0$ and let $(H)$ hold. Assume that

(1) $S(0)=0, D S(0)$ is compact and strongly positive;

(2) for some $m \in \mathbb{N}, S^{m}$ is strictly sublinear on $V$, that is, $S^{m}(\alpha u)>\alpha S^{m}(u)$ for all $\alpha \in(0,1)$ and $u \in V$ with $u \gg 0$.

(a) If $r(D S(0)) \leqslant 1$, then $u=0$ is globally asymptotically stable with respect to $V$;

(b) If $r(D S(0))>1$, then there exists a unique positive fixed point $u_{0}$ of $S$ in $V$ and $u_{0}$ is globally asymptotically stable with repect to $V \backslash\{0\}$. 
Proof: For $S^{m}: V \rightarrow V$, since $S(0)=0$ and $D S(0)$ exists, $D S^{m}(0)$ exists and $D S^{m}(0)=(D S(0))^{m}$. Therefore $D S^{m}(0)$ is compact and strongly positive, and, by the Krein-Rutman theorem, $r\left(D S^{m}(0)\right)=(r(D S(0)))^{m}$. From Theorem 2.1 and the uniqueness of the positive fixed point in Lemma 1 it follows that conclusion (b) holds for $S^{m}$. By Lemma 1 and Theorem 2.2 conclusion (a) holds for $S^{m}$.

By the continuity of $S$ and the definition of the stability of a fixed point with repect to $V$ (see, for example, [7, Definition 2.1] or [13, Definition 3.1]), one can easily prove that if $u_{0}=S^{m}\left(u_{0}\right)=S\left(u_{0}\right)$ then the stability of $u_{0}$ for $S^{m}$ implies that for $S$. Moreover, from [7, Lemma 2.2] or [13, Lemma 3.4] it follows that if for some $u \in V$ we have $\lim _{k \rightarrow \infty}\left(S^{m}\right)^{k}(u)=u_{0}$ and $u_{0}$ is a stable fixed point of $S$ with repect to $V$, then $\lim _{n \rightarrow \infty} S^{n}(u)=u_{0}$. Now conclusions (a) and (b) for $S$ follow from Lemma 2.

This completes the proof.

As an application of Theorem 2.3, we prove the following result.

Theorem 2.4. Let $V=P$ and let $(H)$ hold. Assume that

(1) there exists $b \gg 0$ such that for any $u \in P, \gamma^{+}(u) \cap[0, b]_{E} \neq \emptyset$, where $\gamma^{+}(u)=\left\{S^{n}(u) ; n \in \mathbb{N}\right\}$;

(2) $S(0)=0, D S(0)$ is compact and strongly positive;

(3) $S$ is strictly sublinear on $P$.

Then the conclusions of Theorem 2.3 are valid for $V=P$.

Proof: For any $u>0$, since $b \gg 0$, there exists a sufficiently large $t=t(u)>1$ such that $u \in[0, t b]$. From assumption (1) it follows that there exists $n=n(t) \in \mathbb{N}$ such that $0 \leqslant S^{n}(t b) \leqslant b<t b$. By assumption (3), $S^{n}$ is also strictly sublinear. In the case $r(D S(0))>1$, by applying Theorem 2.3 to $S^{n}: V=[0, t b] \rightarrow V$, we conclude that there exists a stable positive fixed point $u_{0}$ of $S^{n}$ and $\lim _{k \rightarrow \infty}\left(S^{n}\right)^{k}(u)=u_{0}$. By Lemma 2 and the conclusion in the proof of Theorem 2.3, $u_{0}$ is also a stable positive fixed point of $S$ and $\lim _{k \rightarrow \infty} S^{k}(u)=u_{0}$. Now from the uniqueness of the positive fixed point, which is guaranteed by Lemma 1 , it follows that $u_{0}$ is independent of the choice of $u \in P \backslash\{0\}$. Therefore $u_{0}$ is globally asymptotically stable with repect to $P \backslash\{0\}$. In the case $r(D S(0)) \leqslant 1$, a similar proof shows that the other conclusion of Theorem 2.3 holds for $V=P$.

This completes the proof.

REMARK 2.1. If we replace assumptions (1) and (3) in Theorem 2.4 by the following ones:

$(1)^{\prime}$ there exists $b \gg 0$ such that for any $u \in P$, the orbit $\gamma^{+}(u)$ of $u$ ultimately lies in $[0, b]_{E}$, that is, there exists $N=N(u)$ such that for all $n \geqslant N, S^{n}(u) \in[0, b]_{E}$; 
$(3)^{\prime}$ for some $m \in \mathbb{N}, S^{m}$ is strictly sublinear on $P$ respectively, the corresponding conclusions are also valid. To prove this, it suffices to notice that with (1)' one can choose $n$ in the proof of Theorem 2.4 to be such that $n=m \cdot k$ and $k=k(t) \in \mathbb{N}$.

REMARK 2.2. Theorems 2.3 and 2.4 can be viewed as generalisations of $[14$, Theorem 6.1] and [22, Theorems 2.1-2.3] for strongly order-preserving mappings on the finite dimensional ordered space $\left(R^{n}, R_{+}^{n}\right)$ to the case of the infinite dimensional ordered Banach spaces $(E, P)$. Moreover, the crucial concavity hypothesis $(C)$ in [22], that is, $D T(v)-D T(u)>0$ if $u \gg v \gg 0$ (in [14], even $u>v \geqslant 0$ ), implies our strict sublinearity. Indeed, for any $0<\alpha<1, u \gg 0$,

$$
\begin{aligned}
T(\alpha u) & =T(0)+\alpha \int_{0}^{1} D T(s \alpha \cdot u) u \cdot d s \\
& >T(0)+\alpha \int_{0}^{1} D T(s u) u \cdot d s \quad(\text { by }(\mathrm{C})) \\
& =(1-\alpha) T(0)+\alpha T(u) \geqslant \alpha T(u) .
\end{aligned}
$$

In [22, Theorem 2.3], the strong concavity of $T$ as formulated by Krasnoselskii [18], that is, for every $u \gg 0$ and $\alpha \in(0,1)$ there exists $\eta=\eta(u, \alpha)>0$ such that $T(\alpha u) \geqslant(1+\eta) \alpha T(u)$, is assumed. Obviously, this strong concavity implies our strict sublinearity. We further point out that in Theorems 2.1-2.4, we only require the (Fréchet) differentiability of $S$ at $u=0$ (and $u=b$ ).

REMARK 2.3. In [25], Takác introduced the notion of sublinearity of $S$ on $V \subset E$, that is, for any $\alpha \in[0,1], u \in V, S(\alpha u) \geqslant \alpha S(u)$, and studied the convergence of orbits in $V$ and the stability of fixed points of $S$ (see also [13, Section I.5] for a new proof). Our Theorems 2.3 and 2.4 here give a threshold type and much stronger results under the strict sublinearity assumption. On the other hand, our Theorems 2.1 and 2.2 on the global asymptotic behaviors are for more general strongly order-preserving mappings.

REMARK 2.4. According to the remarks on strongly monotone discrete dynamical systems in [8, Section 5], we can replace the standard assumption $(H)$ in this section by the following weaker one:

(H') $V \subset U$ and $S: V \rightarrow V$ is condensing and $S\left([u, v]_{E}\right)$ is bounded in $E$ for all $u, v \in V$ with $u<v$.

A typical example of a condensing map is the following

$$
S=L+K
$$

where $L$ is Lipschitz continuous with Lipschitz constant $<1$ and $K$ is compact. For the definitions of condensing maps and the related Kuratowski noncompactness measure, we refer to $[8,9,26]$. 


\section{Applications to Periodic Parabolic Equations and Cooperative Systems}

Let $T>0$ be fixed and $\Omega \subset R^{N}(N \geqslant 1)$ be a bounded domain with boundary $\partial \Omega$ of class $C^{2+\theta}(0<\theta \leqslant 1)$. We now consider the scalar periodic-parabolic equations

$$
\begin{cases}\frac{\partial u}{\partial t}+A(t) u=f(x, t, u) & \text { in } \Omega \times(0, \infty) \\ B u=0 & \text { on } \partial \Omega \times(0, \infty) \\ u(\cdot, 0)=u_{0} & \text { in } \Omega\end{cases}
$$

where

$$
A(t)=-\sum_{i, j=1}^{N} a_{i j}(x, t) \frac{\partial^{2}}{\partial x_{i} \partial x_{j}}+\sum_{i=1}^{N} a_{i}(x, t) \frac{\partial}{\partial x_{i}}+a_{0}(x, t)
$$

is uniformly elliptic for each $t \in[0, T], a_{i j}(x, t)(1 \leqslant i, j \leqslant N), a_{i}(x, t)(1 \leqslant i \leqslant N)$ and $f(x, t, u)$ are $T$-periodic in $t, B u=u$ or $B u=\frac{\partial u}{\partial v}+b_{0}(x) u$, where $\frac{\partial}{\partial v}$ denotes differentiation in the direction of the outward normal and $b_{0}(x) \geqslant 0$. Let $Q_{T}=$ $\Omega \times[0, T]$. We suppose that

$\left(H_{1}\right) a_{j k}=a_{k j}$ and $a_{i} \in C^{\theta, \theta / 2}\left(\bar{Q}_{T}\right)(1 \leqslant j, k \leqslant N, 0 \leqslant i \leqslant N)$, and $b_{0} \in$ $C^{1+\theta}(\partial \Omega, R)$;

$\left(H_{2}\right) f \in C\left(\bar{Q}_{T} \times R, R\right), \frac{\partial f}{\partial u}$ exists and $\frac{\partial f}{\partial u} \in C\left(\bar{Q}_{T} \times R, R\right)$ with $f(\cdot, \cdot, u)$ and $\frac{\partial f}{\partial u}(\cdot, \cdot, u) \in C^{\theta, \theta / 2}\left(\bar{Q}_{T}, R\right)$ uniformly for $u$ in bounded subsets of $R$.

Let $X=L^{p}(\Omega), N<p<\infty$, and for $\beta \in(1 / 2+N /(2 p), 1]$, let $X_{\beta}$ be the fractional power space of $X$ with respect to $(A(0), B)$ (see [12]). Then $X_{\beta}$ is an ordered Banach space with order cone consisting of the nonnegative functions. Moreover

$$
\begin{gathered}
X_{1}=W_{B}^{2, p}(\Omega) \equiv\left\{u \in W^{2, p}(\Omega) ; B u=0\right\} \\
X_{\beta} \subset C^{1+\lambda}(\bar{\Omega}) \quad \text { with continuous inclusion for } \lambda \in\left[0,2 \beta-1-\frac{N}{p}\right),
\end{gathered}
$$

and the order cone in $X_{\beta}$ has nonempty interior. From [13, Section III.20], it follows that for every $u_{0} \in X_{\beta}$, there exists a unique regular solution $\varphi\left(t, u_{0}\right)$ of (3.1) with the maximal existence interval $I^{+}\left(u_{0}\right) \subset[0, \infty)$ and $\varphi\left(t, u_{0}\right)$ is globally defined provided there is an $L^{\infty}$. bound on $I^{+}\left(u_{0}\right)$.

Let $E=X_{\beta}$ with $\beta \in(12+N /(2 p), 1]$ and assume that there exists an open subset $U$ of $E$ such that for every $u \in U, \varphi(t, u)$ is $L^{\infty}$-bounded on $I^{+}(u)$ x. Then $I^{+}(u)=+\infty$. We define the Poincaré operator $S: U \rightarrow E$ by

$$
S(u)=\varphi(T, u)
$$


From a similar argument to that of [13, Proposition 21.2] or [7, Section 5] it follows that $S: U \rightarrow E$ is continuous, compact and strongly order-preserving. Obviously, a fixed point $u_{0}$ of $S$ corresponds to the $T$-periodic solution $\varphi\left(t, u_{0}\right)$ of (3.1).

Let $u$ be a $T$-periodic solution of (3.1). Consider the corresponding linear periodicparabolic equation

$$
\left\{\begin{array}{l}
\frac{\partial v}{\partial t}+A(t) v=\frac{\partial f}{\partial u}(x, t, u(t, x)) v \\
B v=0
\end{array}\right.
$$

That is,

$$
\left\{\begin{array}{l}
\frac{\partial v}{\partial t}+\bar{A}(t) v=0 \\
B v=0
\end{array}\right.
$$

where $\bar{A}(x, t) v=A(x, t) v-\frac{\partial f}{\partial u}(x, t, u(t, x)) v$. According to [13, Chapter II], (3.2) admits a evolution operator $\bar{U}(t, \tau)(0 \leqslant \tau \leqslant t \leqslant T)$ and for any $0 \leqslant \tau<t \leqslant T, \bar{U}(t, \tau)$ is a compact and strongly positive operator on $E=X_{\beta}$. By [13, Proposition 23.1], the Poincaré operator $S$ associated with (3.1) is defined in a neighbourhood of $u_{0}=u(0)$ and is Fréchet differentiable at $u_{0}$, with $D S\left(u_{0}\right)=\bar{U}(T, 0)$. Let $r=r\left(D S\left(u_{0}\right)\right)$. Then by $[13$, Proposition 14.4], $\mu=(-1 / T) \log (r)$ is the unique principal eigenvalue of the periodic-parabolic eigenvalue problem

$$
\left\{\begin{array}{l}
\frac{\partial v}{\partial t}+\bar{A}(t) v=\mu v \\
B v=0 \\
v \quad T \text {-periodic. }
\end{array}\right.
$$

For various properties and estimates of the principal eigenvalue of linear periodicparabolic problems, we refer to [13,Sections II.15 and 17].

In what follows, we shall impose the following conditions on $f$.

$\left(A_{1}\right) f(x, t, 0) \geqslant 0$ and for every $(x, t) \in \bar{\Omega} \times R, f(x, t, \cdot)$ is sublinear on $I \subset[0, \infty)$, that is, $f(x, t, \alpha u) \geqslant \alpha f(x, t, u)$ for any $\alpha \in(0,1)$ and $u \in I$ with $u>0$, and for at least one $\left(x_{0}, t_{0}\right) \in \Omega \times R, f\left(x_{0}, t_{0}, \cdot\right)$ is strictly sublinear, that is, $f\left(x_{0}, t_{0}, \alpha u\right)>\alpha f\left(x_{0}, t_{0}, u\right)$ for any $\alpha \in(0,1)$ and $u \in I$ with $u>0$.

( $\left.A_{2}\right) f(\cdot, \cdot, 0) \equiv 0$ and for every $(x, t) \in \bar{\Omega} \times R$ and all $u>0$,

$$
f(x, t, u) \leqslant \frac{\partial f(x, t, 0)}{\partial u} \cdot u
$$

for at least one $\left(x_{0}, t_{0}\right) \in \Omega \times R$ and all $u>0$,

$$
f\left(x_{0}, t_{0}, u\right)<\frac{\partial f\left(x_{0}, t_{0}, 0\right)}{\partial u} \cdot u .
$$


Let $P$ be the cone of $E=X_{\beta}$ and

$$
V=\{u \in U ; u(x) \geqslant 0 \text { and } u(x) \in I \text { for all } x \in \bar{\Omega}\}
$$

By the strong positivity of the evolution operator $U(t, \tau)$ on $E$ for $0 \leqslant \tau<t \leqslant T$ and the formula of the variants of constant for the inhomogeneous linear evolution equation, it is not difficult to prove that $\left(A_{1}\right)$ implies the strict sublinearity of the Poincaré operator $S$ on $V$ and $\left(A_{2}\right)$ implies $S(u)<D S(0) u$ for any $u \in V$ with $u \gg 0$. (In the case $f(x, t, 0) \equiv 0$, for any $u \in E$, let $\varphi_{0}(t, u)=\bar{U}(t, 0) u$ be the regular solution of (3.2) with $u_{0}=0$; then $\varphi_{0}(T, u)=D S(0) u$.)

In biological and chemical reaction models, we often encounter the Kolmogorov type reaction function $f$, that is, $f(x, t, u)=u F(x, t, u)$. Let $\mu=\mu(A(t), F(x, t, 0))$ be the principal eigenvalue of the periodic-parabolic problem

$$
\left\{\begin{array}{l}
\frac{\partial v}{\partial t}+A(t) v=F(x, t, 0) v+\mu v \\
B v=0 \\
v \quad T \text {-periodic. }
\end{array}\right.
$$

Then we have the following results.

THEOREM 3.1. Let $f(x, t, u)=u F(x, t, u)$ and let assumptions $\left(H_{1}\right)$ with $a_{0} \geqslant 0$ and $\left(H_{2}\right)$ hold. Assume that

(1) there exists $K_{0}>0$ such that $F(x, t, u)<0$ for all $(x, t) \in \bar{\Omega} \times[0, T]$ and $u \geqslant K_{0}$

(2) $\mu(A(t), F(x, t, 0))<0$.

Then there exist two positive $T$-periodic solutions $u_{1}(t) \leqslant u_{2}(t)$ of (3.1) such that for any solution $u(t)$ of (3.1) with $u(0) \in X_{\beta}$ and $u(0)>0$,

$$
\lim _{t \rightarrow \infty} \operatorname{dist}_{X_{\beta}}\left(u(t),\left[u_{1}(t), u_{2}(t)\right]\right)=0
$$

and (3.1) admits at least one stable positive $T$-periodic solution $u_{0}(t)$ with $u_{1}(t) \leqslant$ $u_{0}(t) \leqslant u_{2}(t)(t \in[0, T])$.

ProOF: By (1), any constant $K \geqslant K_{0}$ is a supersolution of (3.1), and hence for every $u_{0} \in E=X_{\beta}$ with $u_{0}>0$, the solution $\varphi\left(t, u_{0}\right)$ of (3.1) exists globally on $I^{+}\left(u_{0}\right)=[0, \infty)$. Let $S: u_{0} \rightarrow \varphi\left(T, u_{0}\right)$ be the associated Poincaré operator. From assumption (1) it follows that any possible nonnegative $T$-periodic solution $u(t, x)$ satisfies $0 \leqslant u(t, x)<K_{0}$, and hence, by a standard iteration argument for $S$, for any $u_{0} \in E$ with $u_{0} \geqslant 0$ there exists $N=N\left(u_{0}\right)>0$ such that for $n \geqslant N$,

$$
0 \leqslant S^{n}\left(u_{0}\right) \leqslant K_{0} \text { on } \bar{\Omega}
$$


According to [6, Section 2] or [13, Chapter III.21], we may assume, without loss of generality, $K_{0} \in E$. Now the conclusion of the theorem follows from applying Theorem 2.1 with $V=\left[0, K_{0}\right]$ to $S$.

THEOREM 3.2. Let $f(x, t, u)=u F(x, t, u)$ and let $\left(H_{1}\right)$ and $\left(H_{2}\right)$ hold. Assume that

(1) for any $(x, t) \in \bar{\Omega} \times[0, T]$ and any $u>0, F(x, t, u) \leqslant F(x, t, 0)$, and for at least one $\left(x_{0}, t_{0}\right) \in \Omega \times[0, T]$ and any $u>0, F\left(x_{0}, t_{0}, u\right)<F\left(x_{0}, t_{0}, 0\right)$;

(2) $\mu(A(t), F(x, t, 0)) \geqslant 0$.

Then $u=0$ is globally asymptotically stable with respect to nonnegative initial values in $X_{\beta}$.

Proof: From assumption (1) and the comparison theorem for scalar parabolic equations, it follows that for any $u_{0} \in X_{\beta}$ with $u_{0} \geqslant 0$, the solution $\varphi\left(t, u_{0}\right)$ of (3.1) exists on $[0,+\infty)$. Since (1) implies $\left(A_{2}\right)$, the conclusion follows from applying Theorem 2.2 with $V=P$, the cone of $X_{\beta}$, to the associated Poincaré operator $S$.

Remark 3.1. By noticing that $X_{\beta} \hookrightarrow C^{1+\lambda}(\bar{\Omega})$ for $\lambda \in[0,2 \beta-1-N / p)$ and combining Theorems 3.1 and 3.2, we can get a threshold criterion for the "uniform persistence" and "extinction" of single species population. For the uniform persistence (that is, permanence), we refer to $[\mathbf{2 7}, \mathbf{2 8}]$ for reaction-diffusion systems and to [11] for general infinite dimensional dynamical systems. Furthermore, the following Theorem implies a kind of particular uniform persistence, that is, the global attractivity of a unique positive periodic solution.

TheOREM 3.3. Let $f(x, t, u)=u F(x, t, u)$ and let $\left(H_{1}\right)$ and $\left(H_{2}\right)$ hold. Assume that

(1) for any $(x, t) \in \bar{\Omega} \times[0, T], F(x, t, \cdot)$ is decreasing on $[0, \infty)$, and for at least one $\left(x_{0}, t_{0}\right) \in \Omega \times[0, T], F\left(x_{0}, t_{0}, \cdot\right)$ is strictly decreasing on $(0, \infty)$;

(2) there exists a positive supersolution $\bar{V}$ for the periodic boundary value problem (3.1);

(3) $\mu(A(t), F(x, t, 0))<0$.

Then there exists a positive T-periodic solution $u_{0}(t)$ of $(3.1)$ and $u_{0}(t)$ is globally asymptotically stable with respect to positive initial values in $X_{\beta}$.

Proof: Since for any $\rho \geqslant 1$, by assumption (2), $\rho \bar{V}$ is also a supersolution of (3.1), the global existence of any solution $u(t)$ of (3.1) with nonnegative initial values in $E$ follows. Obviously, assumption (1) implies $\left(A_{1}\right)$ with $I=[0, \infty)$ and hence the strict sublinearity of the associated Poincaré operator $S: P \rightarrow P$, where $P$ is the cone of $E$. By Lemma 1 in Section 2, $S$ admits at most one positive fixed point in $P$. Without loss of generality, we may assume that $\bar{V}(0) \in E=X_{\beta}$ (see [6, Section 2] 
or $\left[13\right.$, Chapter III.21\}). On applying Theorem 2.3 with $V=[0, \rho \bar{V}(0)]_{E} \subset P$ for any $\rho \geqslant 1$, we then complete the proof of the theorem.

REMARK 3.2. In [13, Chapter III.28], Hess considered the periodic-parabolic Logistic equation, that is,

$$
\begin{cases}\frac{\partial u}{\partial t}+A(t) u=m u-b u^{2} & \text { in } \Omega \times(0, \infty) \\ B u=0 & \text { on } \partial \Omega \times(0, \infty)\end{cases}
$$

where $m$ and $b \in C^{\theta, \theta / 2}(\bar{\Omega} \times R)$ and are $T$-periodic in $t$, and $b(x, t) \geqslant 0, b(x, t) \not \equiv 0$ on $\bar{Q}_{T}$. Let $F(x, t, u)=m(x, t)-b(x, t) u$. Obviously, both assumptions (1) of Theorems 3.2 and 3.3 are satisfied, and hence Theorems 3.2 and 3.3 imply [13, Theorem 28.1].

Now we turn to the case that (3.1) is autonomous, that is, $A(x, t)=A(x)$ and $F(x, t, u)=F(x, u)$. We distinguish between two cases:

(I) $a_{0}(x) \geqslant 0$, with $a_{0}(x) \not \equiv 0$ if $b_{0}(x) \equiv 0$;

(N) $a_{0}(x) \equiv 0, b_{0}(x) \equiv 0$.

In case (I), we assume $m \in C^{\theta}(\bar{\Omega})$ and $m(x)>0$ at some $x \in \bar{\Omega}$. From [13, Theorem 16.1 and Remark 16.5] it follows that the elliptic eigenvalue problem

$$
\begin{cases}A(x) u=\lambda m(x) u & \text { in } \Omega \\ B u=0 & \text { on } \partial \Omega\end{cases}
$$

has a unique positive principal eigenvalue $\lambda_{1}(m)$. For any $T>0$, let $\mu(A, m(x), T)$ be the principal eigenvalue of the periodic-parabolic problem (3.4) with $F(x, t, 0)$ replaced by $m(x)$. From [13, Chapter II.15 and Remark 16.5] it follows that if $\lambda_{1}(m)<1$, then $\mu(A, m(x), T)<0$, and if $\lambda_{1}(m) \geqslant 1$, then $\mu(A, m(x), T) \geqslant 0$. As a corollary of Theorems 3.2 and 3.3 , we have the following result.

THEOREM 3.4. Let $A(x, t)=A(x), f(x, t, u)=u F(x, u)$ and let $\left(H_{1}\right),\left(H_{2}\right)$ and $(I)$ hold. Assume that

(1) $F(x, 0)>0$ at some $x \in \Omega$;

(2) for any $x \in \bar{\Omega}, F(x, \cdot)$ is decreasing on $[0, \infty)$, and for at least one $x_{0} \in \Omega$, $F\left(x_{0}, \cdot\right)$ is strictly decreasing on $(0, \infty)$;

(3) there exists a positive supersolution $\bar{V}$ for the corresponding steady-state problem

$$
\begin{cases}A(x) u=u F(x, u) & \text { in } \Omega \\ B u=0 & \text { on } \partial \Omega .\end{cases}
$$


(a) If $\lambda_{1}(F(x, 0)) \geqslant 1$, then (3.6) has no positive solution in $X_{\beta}$ and $u=0$ is a globally asymptotically stable solution of (3.1) with repect to nonnegative initial values in $X_{\beta}$;

(b) If $\lambda_{1}(F(x, 0))<1$, then (3.6) has a unique positive solution $u_{0}$ in $X_{\beta}$ and $u=u_{0}$ is a globally asymptotically stable solution of (3.1) with respect to positive initial values in $X_{\beta}$.

Proof: For given $T>0$, we view the autonomous parabolic equation (3.1) as a $T$-periodic one. From Theorem 3.2 the first conclusion (a) follows. In the second case, by Theorem 3.3, (3.1) has a unique positive $T$-periodic solution $u_{0}(t, x)$ and $u_{0}(t, x)$ is globally asymptotically stable. For any $\gamma>0$, since (3.1) is autonomous, $u_{0}(t+\gamma, x)$ is also a $T$-periodic solution of (3.1). By the uniqueness of the positive $T$-periodic solution, $u_{0}(t+\gamma, x) \equiv u_{0}(t, x)$ for all $t \in[0, T], x \in \Omega$. This implies that $u_{0}(t, x) \equiv u_{0}(0, x), x \in \Omega$, that is, $u_{0}$ is a steady-state solution of $(3.1)$, and hence the second conclusion follows.

This completes the proof.

REMARK 3.3. It is obvious that the following assumption

$(3)^{\prime}$ there exists $K_{0}>0$ such that for all $x \in \bar{\Omega}, \quad F\left(x, K_{0}\right) \leqslant 0$

implies condition (3) in Theorem 3.4. Similar results to the second conclusion of Theorem 3.4 were proved for diffusive Logistic equation under condition (3)' and Dirichlet boundary condition (see [3, Theorems 2.1 and 2.3] and [4, Theorem 2.4 with Corollary 3.3]). For the biological interpretation and significance of these results, we further refer to $[3,4]$.

For the case (N), according to [13, Theorem 16.3 and Remark 16.5], we can also discuss the global asymptotic stability of the steady-state solution of the corresponding autonomous equation (3.1) in a similar way.

EXAMPLE 1. We consider a reaction-diffusion equation of a single population growth, which is deduced from a competition model in an unstirred chemostat (see $[\mathbf{1 7}, \mathbf{2 4}]$ ).

$$
\left\{\begin{array}{l}
\frac{\partial^{2} u}{\partial x^{2}}+F(\phi(x)-u) u, \quad t>0,0<x<1 \\
\frac{\partial u}{\partial x}(t, 0)=0, \frac{\partial u}{\partial x}(t, 1)+\gamma u(t, 1)=0 \\
u(0, x)=u_{0}(x) \geqslant 0, \quad \text { and } u_{0}(x) \leqslant \phi(x), x \in(0,1)
\end{array}\right.
$$

where $d>0, \phi(x)=S^{(0)}((1+\gamma) / \gamma-x), 0<x<1, S^{(0)}>0, \gamma>0$ and $F$ is the typical Michaelis-Menten- Monod response function:

$$
F(s)=\frac{m s}{a+s}, \quad s \geqslant 0 \quad(m>0, a>0)
$$


In what follows, we consider a more general function $F(s)$ satisfying

$$
F(0)=0 \text { and } F^{\prime}(s)>0 \text { for } s \geqslant 0
$$

Let $\lambda_{0}=\lambda_{0}(F(\phi(x)))>0$ be the first eigenvalue of

$$
\left\{\begin{array}{l}
d \frac{d^{2} v}{d x^{2}}+\lambda F(\phi(x)) v=0 \\
v^{\prime}(0)=0, v^{\prime}(1)+\gamma v(1)=0
\end{array}\right.
$$

Then we have the following result.

Proposition. Assume (3.8) holds.

(a) If $\lambda_{0}(F(\phi(x))) \geqslant 1$, then $u=0$ is a globally asymptotically stable steadystate solution of (3.7) with respect to nonnegative initial values.

(b) If $\lambda_{0}(F(\phi(x)))<1$, then (3.7) has a globally asymptotically stable positive steady-state solution $u_{0}(x)\left(u_{0}(x)<\phi(x)\right), x \in(0,1)$ with respect to positive initial values.

Proof: For the use of Theorem 3.4, let $\widehat{F}(s), s \in R$ be any continuously differentiable extension of $F(s)$ on $[0, \infty)$ to $R$ satisfying $\hat{F}^{\prime}(s)>0$ for all $s \in R$.

Consider autonomous parabolic equation

$$
\left\{\begin{array}{l}
\frac{\partial u}{\partial t}=d \frac{\partial^{2} u}{\partial x^{2}}+\widehat{F}(\phi(x)-u) u, \quad t>0,0<x<1 \\
\frac{\partial u}{\partial x}(t, 0)=0, \quad \frac{\partial u}{\partial x}(t, 1)+\gamma u(t, 1)=0 \\
u(0, x)=u_{0}(x) \geqslant 0
\end{array}\right.
$$

Let $K_{0}=S^{(0)} \cdot(1+\gamma) / \gamma$. Then $\phi(x) \leqslant K_{0}, x \in(0,1)$ and hence $\widehat{F}\left(\phi(x)-K_{0}\right) \leqslant 0$. Then Theorem 3.4 implies the corresponding conclusion for (3.10). From a comparison argument it easily follows that for any $0 \leqslant u_{0}(x) \leqslant \phi(x)$, the solution $u(t, x)$ of (3.10) satisfies $0 \leqslant u(t, x) \leqslant \phi(x), t \geqslant 0$, and hence the conclusion for (3.7) follows.

REMARK 3.4. In [17], Hsu and Waltman considered (3.7) with $F=\left(m_{i} s\right) /\left(a_{i}+s\right)$ $(s \geqslant 0)(i=u, v)$. Obviously, the above Proposition implies [17, Theorems 3.1 and 3.2]. Moreover, our Proposition also includes the critical case $\lambda_{0}(F(\phi(x)))=1$.

Finally, we consider the periodic system of ordinary differential equations

$$
\left\{\begin{array}{l}
\frac{d x}{d t}=F(t, x) \\
x(0)=x_{0} \in R_{+}^{n}
\end{array}\right.
$$


where $x=\left(x_{1}, \ldots, x_{n}\right) \in R^{n}$ and $R_{+}^{n}=\left\{x \in R^{n} ; x_{i} \geqslant 0, i=1,2, \ldots, n\right\}$. We assume that $F: R_{+}^{1} \times R_{+}^{n} \rightarrow R^{n}$ is continuous and $T$-periodic in $t$, and that all partial derivatives $\frac{\partial F_{i}}{\partial x_{j}}(1 \leqslant i, j \leqslant n)$ exist and are continuous in $R_{+}^{1} \times R_{+}^{n}$.

In what follows, we take $R^{n}$ as an ordered Banach space with its natural cone $R_{+}^{n}$ and denote the interior of $R_{+}^{n}$ by $\dot{R}_{+}^{n}$. An $n \times n$ matrix $A$ is said to be quasipositive if its off-diagonal entries are nonnegative. It is irreducible if viewed as a linear mapping from $R^{n}$ to $R^{n}$, it does not leave invariant any proper linear subspace spanned by a subset of the standard basis vectors of $R^{n}$. For the other equivalent definitions of irreducibility, we refer to $[\mathbf{2}, \mathbf{1 5}]$.

We first impose the following conditions on $F(t, u)$.

(C1) $F_{i}(t, x) \geqslant 0$ for every $x \geqslant 0$ with $x_{i}=0, t \in R_{+}^{1},(i=1,2, \ldots, n)$;

(C2) $\frac{\partial F_{i}}{\partial x_{j}} \geqslant 0, i \neq j$, for all $(t, x) \in R_{+}^{1} \times R_{+}^{n}$, and $D_{x} F(t, x)=\left(\frac{\partial F_{i}}{\partial x_{j}}\right)_{1 \leqslant i, j \leqslant n}$ is irreducible for each $t \in R_{+}^{1}, x \in R_{+}^{n}$.

Assume (C1) and C(2) hold. Then for every $x \in R_{+}^{n}$, there exists a unique solution $\varphi(t, x)$ of (3.11) with the maximal existence interval $I^{+}(x) \subset[0,+\infty)$ and $\varphi(t, x) \geqslant$ $0\left(t \in I^{+}(x)\right)$. If there exists a relatively open and convex subset $U$ of $R_{+}^{n}$ such that for every $x \in U, \varphi(t, x)$ is bounded on $I^{+}(x)$, then $I^{+}(x)=+\infty$. We can define the Poincaré operator $S: U \rightarrow R_{+}^{n}$ by

$$
S(u)=\varphi(T, u), u \in U
$$

From a Kamke's theorem argument it follows that $S: U \rightarrow R_{+}^{n}$ is strongly orderpreserving (for example, see [15, Theorem 1.5]). Now let $x(t)$ be a nonnegative $T$ periodic solution of (3.11) and consider the corresponding linear periodic systems

$$
\frac{d z}{d t}=D_{x} F(t, x(t)) z
$$

By (C2), $A(t) \equiv D_{x} F(t, x(t))$ is a continuous, $T$-periodic, quasipositive and irreducible matrix function. Let $\phi(t)$ be the fundamental matrix solution of (3.12) with $\phi(0)=I$ (the identity matrix). By [2, Lemma 2] or [15, Theorem 1.1], for each $t>0, \phi(t)$ is a matrix with all entries positive, and hence for each $t>0, \phi(t): R^{n} \rightarrow R^{n}$ is a compact and strongly positive linear operator. From the continuity and differentiability of solutions for initial values, it easily follows that the Poincaré operator $S$ associated with (3.11) is defined in a neighbourhood of $x_{0}=x(0)$ and differentiable at $x_{0}$, with $D S\left(x_{0}\right)=\phi(T)$. The eigenvalues of $\phi(T)$ are often called the Floquet multipliers of (3.12). Based on the Krein-Rutman theorem (or on the Perron-Frobenius theorem in our present finite dimensional case), we call $\rho=r(\phi(T))$ the principal Floquet multiplier of (3.12). 
In what follows, we shall further impose the following conditions on $F$.

(C3) $F(t, \cdot)$ is strictly sublinear on $\dot{R}_{+}^{n}$ for each $t \in R_{+}^{1}$, that is, for each $x \gg 0$ and $0<\alpha<1$,

$$
F(t, \alpha x)>\alpha F(t, x), t \geqslant 0
$$

(C4) $F(t, 0) \equiv 0$ and for every $t \in R_{+}^{1}$ and $x \gg 0$,

$$
F(t, x)<D_{x} F(t, 0) x \text {. }
$$

Let $A(t)(t \geqslant 0)$ be a continuous, quasipositive and irreducible matrix function, and $\phi(t, \tau)(t \geqslant \tau \geqslant 0)$ be the fundamental matrix solution of $\frac{d x}{d t}=A(t) x$ with $\phi(\tau, \tau)=$ I. From the proof of [2, Lemma 2] or [15, Theorem 1.1] it follows that for each $t>\tau, \phi(t, \tau): R^{n} \rightarrow R^{n}$ is strongly positive linear operator. Now by the formula for the variation of constant for the inhomogeneous linear ordinary differential equation, one can easily prove that (C3) implies the strict sublinearity of the Poincare operator on $R_{+}^{n}$ and (C4) implies $S(x)<D S(0) x$ for any $x \gg 0$. Therefore, by applying Theorems in Section 2 to the Poincaré operator defined by (3.11), we can formulate the corresponding theorems for (3.11). As an illustration of Theorem 2.4, we have the following result.

Theorem 3.5. Let (C1), (C2) and (C3) hold. Assume that $F(t, 0) \equiv 0$ and there exists a bounded subset $B$ of $R_{+}^{n}$ such that any solution $x(t)$ of (3.11) ultimately lies in $B$. Let $\rho$ be the principal Floquet multiplier of (3.12) with $x(t) \equiv 0$.

(a) If $\rho \leqslant 1$, then $x(t) \equiv 0$ is a globally asymptotically stable periodic solution of (3.11) with repect to the initial values in $R_{+}^{n}$;

(b) If $\rho>1$, then (3.11) has a unique positive $T$-periodic solution $x(t)$ and $x(t)$ is globally asymptotically stable with respect to initial values in $R_{+}^{n} \backslash\{0\}$.

REMARK 3.5. In a similar way to the parabolic equations, from Theorem 3.5 we can easily deduce an analogous conclusion for autonomous systems (3.11), that is, for $F(t, u)=F(u)$.

EXAMPLE 2. Consider the single loop positive feedback systems in $R_{+}^{n}$ (see $\left.[22,25]\right)$ :

$$
\left\{\begin{array}{l}
\frac{d x_{1}}{d t}=f\left(x_{n}, t\right)-\alpha_{1}(t) x_{1} \\
\frac{d x_{2}}{d t}=x_{1}-\alpha_{2}(t) x_{2} \\
\cdots \cdots \cdots \cdots \cdots \cdots \\
\frac{d x_{n}}{d t}=x_{n-1}-\alpha_{n}(t) x_{n}
\end{array}\right.
$$


Assume that $\alpha_{i}(\cdot)$ and $f\left(x_{n}, \cdot\right)$ are continuous and $T$-periodic in $t \in R_{+}^{1}$, that $f(0, t) \equiv 0, f(u, t) \geqslant 0, \frac{\partial f}{\partial u}(u, t)>0$ is continuous in $R_{+}^{2}$, and that for each $t \in R_{+}^{1}$, $f(\cdot, t)$ is strictly sublinear on $R_{+}^{1}$, that is, for any $t \geqslant 0, u>0$ and $0<\alpha<1$, $f(\alpha u, t)>\alpha f(u, t)$. It is easy to verify that (C1), (C2) and (C3) are satisfied for (3.13). If we further assume that

$$
\begin{gathered}
f(u, t) \leqslant a u+b, \quad a, b \geqslant 0, \\
\alpha_{i}=\min _{0 \leqslant t \leqslant T} \alpha_{i}(t)>0,1 \leqslant i \leqslant n, \\
a<\prod_{i=1}^{n} \alpha_{i},
\end{gathered}
$$

then the ultimate boundedness of (3.13) follows from that of a nonhomogeneous linear system which majorises (3.13) (for some details, see [22]). Therefore Theorem 3.5 now applies to (3.13). A similar result was proved in $[22]$ with $f(\cdot, t)$ strongly concave.

\section{REFERENCES}

[1] H. Amann, 'Fixed point equations and nonlinear eigenvalue problems in ordered Banach spaces', SIAM Rev. 18 (1976), 620-709.

[2] G. Aronsson and R.B. Kellogg, 'On a differential equation arising from compartmental analysis', Math. Biosci. 38 (1973), 113-122.

[3] R.S. Cantrell and C. Cosner, 'Diffusive logistic equations with indefinite weights: population models in disrupted envionments', Proc. Roy. Soc. Edinburgh Sect. A 112 (1989), 293-318.

[4] R.S. Cantrell and C. Cosner, 'Diffusive logistic equations with indefinite weights: population models in disrupted envionments II', SIAM J. Math. Anal. 22 (1991), 1043-1064.

[5] E.N. Dancer, 'Fixed point index calculations and applications', in Topological nonlinear analysis, degree, singularity and variation, (M. Matzeu and A. Vignili, Editors) (Birkhauser, 1995).

[6] E.N. Dancer and P. Hess, 'On stable solutions of quasilinear periodic-parabolic problems', Ann. Scuola Norm. Sup. Pisa Cl. Sci. 14 (1987), 123-141.

[7] E.N. Dancer and P. Hess, 'Stability of fixed points for order-preserving discrete-time dynamical systems', J. Reine Angew. Math. 419 (1991), 125-139.

[8] D. Daners, 'Qualitative behavior of an epidemics model', Differential Integral Equations 5 (1992), 1017-1032.

[9] J.K. Hale, Asymptotic behavior of dissipative systems, Math. Surveys Monographs 25 (Amer. Math. Soc., Providence, RI, 1988).

[10] J.K. Hale and G. Rangel, 'Convergence in gradient-like systems with appllications to PDE', Z. Angew. Math. Phys. 43 (1992), 63-124. 
[11] J.K. Hale and P. Waltman, 'Persistence in infinite dimensional systems', SIAM J. Math. Anal. 20 (1989), 388-395.

[12] D. Henry, Geometric theory of semilinear parabolic equations, Lecture Notes in Math. 840 (Springer-Verlag, Berlin, 1981).

[13] P. Hess, Periodic-parabolic boundary value problems and positivity, Pitman Research Notes in Math. 247 (Longman Scientific and Technical, 1991).

[14] M.W. Hirsch, 'The dynamical systems approach to differential equations', Bull. Amer. Math. Soc. 11 (1984), 1-64.

[15] M. W. Hirsch, 'Systems of differential equations that are competitive or cooperative II: convergence almost everywhere', SIAM J. Math. Anal. 16 (1985), 423-439.

[16] M.W. Hirsch, 'Stability and convergence in strongly monotone dynamical systems', $J$. Reine Angew. Math. 383 (1988), 1-53.

[17] S.-B. Hsu and P. Waltman, 'On a system of reaction-diffusion equations arising from competition in an unstirred Chemostat', SIAM J. Appl. Math. 53 (1993), 1026-1044.

[18] M.A. Krasnoselskii, Translation along trajectories of differential equations, Translations of Math. Monographs 19 (Amer. Math. Soc., Providence, R.I., 1968).

[19] H. Matano, 'Existence of nontrivial unstable sets for equilibriums of strongly order-preserving systems', J. Fac. Sci. Univ. Tokyo Sect. IA Math. 30 (1984), 645-673.

[20] P. Poláčik, 'Convergence in smooth strongly monotone flows defined by semilinear parabolic equations', J. Differential Equations 79 (1989), 89-110.

[21] P. Poláčik and I. Tereščák, 'Convergence to cycles as a typical asymptotic behavior in smooth strongly monotone discrete-time dynamical systems', Arch. Rational Mech. Anal. 116 (1991), 339-360.

[22] H. L. Smith, 'Cooperative systems of differential equations with concave nonlinearities', Nonlinear Anal. 10 (1986), 1037-1052.

[23] H.L. Smith and H.R. Thieme, 'Convergence for strongly order-preserving semiflows', SIAM J. Math. Anal. 22 (1991), 1081-1101.

[24] J. So and P. Waltman, 'A nonlinear boundary value problem arising from competition in the Chmostat', Appl. Math. Comput. 32 (1989), 169-183.

[25] P. Takáć, 'Asymptotic behavior of discrete-time semigroups of sublinear, strongly increasing mappings with applications in biology', Nonlinear Anal. 14 (1990), 35-42.

[26] E. Zeidler, Nonlinear functional analysis and its applications I. Fixed-point theory (Berlin, Heidelberg, New York, 1986).

[27] X.-Q. Zhao and V. Hutson, 'Permanence in Kolmogorov periodic predator-prey models with diffusion', Nonlinear Anal. 23 (1994), 651-668.

[28] X.-Q. Zhao and B.D. Sleeman, 'Permenence in Kolmogorov competition models with diffusion', IMA J. Applied Math. 51 (1993), 1-11.

Institute of Applied Mathematics

Academia Sinica

Beijing 100080

People's Republic of China 